\title{
Cas de fièvre par morsure de rat sur l'île de Vancouver, 2010 à 2016
}

\author{
BN Hryciw ${ }^{1}$, CP Wright ${ }^{1}, \mathrm{~K} \mathrm{Tan}^{1,2 \star}$
}

\section{Résumé}

Contexte : La fièvre par morsure de rat (FMR) est une infection bactérienne zoonotique rare causée par Streptobacillus moniliformis et Spirillum minus, naturellement présents dans les voies respiratoires des rongeurs. Récemment, de multiples cas de FMR ont été observés sur l'île de Vancouver (Colombie-Britannique).

Objectif : Analyser une série de cas de FMR observés sur l'île de Vancouver entre 2010 et 2016 afin de caractériser l'épidémiologie, le tableau clinique, la microbiologie et le traitement de la FMR.

Méthodologie : Les cas ont été recensés grâce à une recherche sur le diagnostic au moment du congé et d'information du laboratoire de microbiologie. Des renseignements cliniques ont été recueillis lors de l'examen des dossiers médicaux électroniques et papier des hôpitaux effectué par Island Health.

Résultats : Onze cas de FMR ont été recensés sur l'île de Vancouver entre 2010 et 2016. La plupart des cas de FMR ont été confirmés par l'identification de $S$. moniliformis par culture ou technique moléculaire. Tous les cas présentaient une fièvre et un sous-ensemble de cas présentait au moins un des symptômes suivants : myalgie, éruption cutanée, polyarthralgie, épanchement articulaire et vomissements. Tous les cas ont été traités avec succès par la pénicilline, la ceftriaxone ou la doxycycline. Sept cas ont nécessité une hospitalisation, mais aucun décès ni aucune morbidité significative n'a été observé.

Conclusion : Il s'agit de la série de cas de FMR la plus importante au Canada. Les cliniciens doivent maintenir un indice de suspicion élevé lors du diagnostic, et une intervention précoce est nécessaire pour prévenir la morbidité et la mortalité.

\section{Affiliations}

${ }^{1}$ Université de la

Colombie-Britannique, Vancouver (Colombie-Britannique)

${ }^{2}$ Agency for Pathology and Laboratory Medicine de la Colombie-Britannique, Vancouver (Colombie-Britannique)

*Correspondance : kennard.tan@ bccss.org

Citation proposée : Hryciw BN, Wright CP, Tan K. Cas de fièvre par morsure de rat sur l'île de Vancouver, 2010 à 2016. Relevé des maladies transmissibles au Canada 2018;44(9);243-7. https://doi.org/10.14745/ccdr. v44i09a05f

Mots-clés : fièvre par morsure de rat, infection zoonotique, Streptobacillus moniliformis, Spirillium minus, résumé graphique

\section{Introduction}

La fièvre par morsure de rat (FMR) est une infection zoonotique rare causée par les bacilles à gram négatif Streptobacillus moniliformis et Spirillium minus (1,2). Ces bactéries font partie de la flore commensale (p. ex. la flore buccale normale) des rats domestiques et de laboratoire en bonne santé (3).

S. moniliformis est l'agent pathogène prédominant en Amérique du Nord, tandis que $S$. minus est plus répandu en Asie, où la FMR est également appelée «sodoku » $(4,5)$. La FMR porte bien son nom, car l'infection se transmet généralement par morsure de rongeurs; cependant, elle peut également se transmettre par griffure ou par contact mucocutané avec la salive, I'urine ou les excréments de rongeurs (4). Historiquement, la population à risque de FMR était limitée au personnel de laboratoire et aux personnes de statut socio-économique inférieur (6). La domestication des rongeurs a mené à l'élargissement de l'épidémiologie de la FMR pour inclure les propriétaires de rongeurs domestiques et les employés d'animaleries $(6,7)$. En raison de la manipulation plus fréquente des rongeurs, une augmentation concomitante des morsures a été observée. Dans un rapport, le nombre estimé de morsures de rat aux États-Unis est de 40000 par année, dont $2 \%$ causent une infection (8). Des études précédentes ont révélé que la FMR constitue un enjeu de santé publique significatif et prenant de l'ampleur; par conséquent, il est nécessaire de mieux comprendre la FMR sur le plan clinique $(5,9)$.

\section{Tableau clinique, diagnostic et traitement}

À la suite de l'inoculation de l'agent pathogène, la période d'incubation de S. moniliformis est habituellement de moins de sept jours, mais varie de trois jours à trois semaines (4). La FMR est généralement caractérisée par des symptômes manifestes, soit la fièvre, les frissons, les éruptions cutanées et la 
polyarthralgie. Les autres symptômes peuvent inclure la fatigue, les vomissements, la myalgie, les céphalées et la pharyngite (10). Comme les symptômes sont non spécifiques et variables, le diagnostic clinique de FMR n'est souvent pas établi si les antécédents d'exposition à des rongeurs ne sont pas connus. Le tableau clinique des infections par $S$. minus est légèrement différent, caractérisé par une induration et une ulcération possible au site de la morsure et par une adénopathie connexe après une incubation de 14 à 18 jours (4). Les complications de la FMR non traitée comprennent l'apparition d'une myocardite, d'une péricardite, d'une méningite, d'une amniotite et d'abcès dans divers organes, ainsi que le décès touchant jusqu'à $13 \%$ des cas (11-14).

Le diagnostic de FMR en laboratoire de microbiologie est souvent difficile à établir et retardé en raison de la nature complexe des bacilles $S$. moniliformis et $S$. minus $(1,4)$. La culture des micro-organismes est lente et leur croissance peut être inhibée par des substances présentes dans le milieu de culture. Heureusement, les micro-organismes sont habituellement sensibles à divers antibiotiques, dont les bêta-lactamines, la clindamycine, l'érythromycine et la tétracycline $(3,4)$. Parmi ceux-ci, le traitement recommandé est la pénicilline (15).

\section{Contexte canadien}

Les signalements de cas de FMR au Canada ont rarement été documentés avant 2002 (16), bien que des cas aient été décrits au Canada dès la première moitié du $20^{\mathrm{e}}$ siècle $(17,18)$. Par la suite, un total de sept cas d'infection par S. moniliformis ont été signalés au Canada, principalement en Ontario et au Québec $(16,19-21)$. Plus récemment en 2013 , un seul cas a été signalé sur l'île de Vancouver (22). En incluant ce cas récent, nous avons observé plusieurs cas confirmés de FMR sur l'île de Vancouver depuis 2010.

L'objectif de cette étude de série de cas est de décrire l'étiologie, l'épidémiologie et les caractéristiques cliniques de la FMR au sein de la population de l'île de Vancouver entre 2010 et 2016. Les objectifs secondaires consistent à examiner l'issue clinique des patients, dont la longueur du séjour à l'hôpital, le traitement administré et les séquelles.

\section{Méthodologie}

\section{Contexte et population}

Island Health est une autorité régionale de santé qui fournit des soins hospitaliers et d'urgence aux 765000 résidents de l'île de Vancouver. Les cas de FMR ont été recensés grâce à I'interrogation du système Enterprise Data Warehouse d'Island Health. Plus précisément, l'interrogation de la Base de données sur les congés des patients au sujet des patients admis selon les codes de diagnostic de la CIM-10-CA (Classification internationale des maladies et des problèmes de santé connexes, dixième révision, Canada) pour la "streptobacillose » (A25.1) ou "fièvre causée par morsure de rat, sans précision » (A25.9), ainsi que le système d'information du laboratoire de microbiologie sur S. moniliformis ou S. minus entre janvier 2010 et décembre 2016. Les patients ayant reçu une confirmation microbiologique de S. moniliformis ou de S. minus, ou ceux ayant été exposés à des rongeurs et présentant un syndrome clinique compatible ont été inclus dans la série de cas. Les données ont été recueillies à partir des examens rétrospectifs des dossiers médicaux des cas recensés concernant les caractéristiques cliniques au moment de la consultation, le traitement administré et les résultats cliniques. Cette étude a exigé et obtenu l'approbation des comités d'éthique de la recherche.

\section{Collecte et analyse des données}

Des renseignements épidémiologiques et cliniques sur les cas recensés ont été recueillis à partir des dossiers médicaux électroniques et papier des hôpitaux. Plus particulièrement, les auteurs ont tenté de compiler, le cas échéant, toute information concernant la source d'exposition, y compris le contexte d'interaction, notamment les morsures, les griffures ou le contact avec des rongeurs domestiques, de laboratoire ou sauvages. Les données sur la population ont été analysées à l'aide de statistiques descriptives afin d'estimer l'épidémiologie, les caractéristiques cliniques et les issues cliniques entre les patients.

\section{Résultats}

Onze cas de FMR ont été recensés sur l'île de Vancouver entre 2010 et 2016 (tableau 1). L'âge médian des patients au moment du diagnostic était de 20 ans, allant de cinq à 57 ans. Les cas ont été divisés sans biais significatif lié au genre, avec cinq hommes et six femmes. Neuf patients ont signalé une exposition à des rongeurs (82\%); il s'agissait de rats domestiques dans tous les cas. Parmi les cinq cas ayant des griffures et/ou des morsures documentées, trois ont fourni la date de la morsure ou de la griffure. Le temps écoulé entre l'exposition et la consultation d'un pourvoyeur de soins de santé était de sept, huit et 19 jours.

Les signes et les symptômes signalés lors de la consultation initiale ont été inclus pour les 11 cas. Une fièvre, au moins intermittente, a été signalée dans tous les cas (100\%). Les signes et les symptômes marqués comprenaient une polyarthralgie dans cinq cas (45\%), des éruptions cutanées dans trois cas (27\%) et des épanchements articulaires dans trois cas (27\%). Les autres symptômes courants comprenaient des vomissements (36 \%), la myalgie (18\%), des frissons (18\%) et la dorsalgie (18\%). Deux cas (18\%) de complications possibles ou avérées ont été signalés : un cas ayant développé une septicémie et l'autre étant soupçonné d'avoir développé un érythème noueux, bien qu'aucune autre donnée n'ait été fournie par la suite.

Dix (91\%) des 11 cas ont été confirmés par un diagnostic microbiologique, et le cas restant a été confirmé par un diagnostic clinique basé sur les antécédents et les symptômes. Des hémocultures ont été réalisées pour tous les cas dans un milieu d'hémoculture standard, milieux BD BACTEC Plus Aerobic et BD BACTEC Lytic Anaerobic (Becton Dickinson, Sparks, Maryland). Huit (73\%) cultures étaient positives à l'égard de S. moniliformis. Deux autres cas ont été recensés en raison de la présence de l'acide ribonucléique (ARN) $16 \mathrm{~S}$ de S. moniliformis dans le milieu d'hémoculture négatif ou à partir d'une aspiration articulaire. Dans un cas d'hémoculture positive, on a également pu identifier S. moniliformis par l'analyse de I'ARN 16S dans un échantillon de liquide provenant d'un épanchement articulaire du genou. En ce qui concerne les quatre cas les plus récents avec 
Tableau 1 : Cas signalés de fièvre par morsure de rats sur l'île de Vancouver de 2010 à 2016

\begin{tabular}{|c|c|c|c|c|c|c|}
\hline $\begin{array}{l}\text { Âge (années) / } \\
\text { sexe }\end{array}$ & $\begin{array}{c}\text { Exposition à des } \\
\text { rats }^{\mathrm{a}} \text {; } \\
\text { voie } \mathrm{d}^{\text {'inoculation }}\end{array}$ & Incubation & $\begin{array}{l}\text { Diagnostic; agent } \\
\text { pathogène }\end{array}$ & $\begin{array}{c}\text { Caractéristiques cliniques } \\
\text { et complications }\end{array}$ & Schéma thérapeutique & $\begin{array}{c}\mathrm{N}^{\text {bre }} \text { de jours } \\
\text { d'antibiothérapie }^{\text {b }}\end{array}$ \\
\hline $5 / \mathrm{H}$ & $+; \mathrm{NS}$ & Inconnue & $\begin{array}{l}\text { Culture -, ARN } 16 \mathrm{~S}+\text {; } \\
\text { S. moniliformis }\end{array}$ & Fièvre, vomissements & Ceftriaxone, pénicilline & $7(4 \mathrm{j} I \mathrm{~V})$ \\
\hline $7 / F$ & $+; \mathrm{NS}$ & Inconnue & Culture -, ARN 16S +; inconnu & $\begin{array}{l}\text { Fièvre, éruption cutanée, } \\
\text { polyarthralgie, vomissements, } \\
\text { épanchement articulaire, } \\
\text { septicémie }\end{array}$ & Gentamicine, pénicilline & $14(7 \mathrm{j} I V)$ \\
\hline $14 / F$ & $\begin{array}{l}+; \text { morsure et } \\
\text { griffure }\end{array}$ & 7 jours & Culture $+;$ S. moniliformis & Fièvre, myalgie, frissons & Ceftriaxone, amoxicilline & $16(6$ j IV) \\
\hline $17 / F$ & NS ; NS & Inconnue & Culture $+;$ S. moniliformis & Fièvre, dorsalgie & Ceftriaxone & Inconnue \\
\hline $17 / H$ & + ; griffure & Inconnue & $\begin{array}{l}\text { Culture +, ARN 16S +; S. } \\
\text { moniliformis }\end{array}$ & $\begin{array}{l}\text { Fièvre, polyarthralgie, } \\
\text { épanchement articulaire }\end{array}$ & Ceftriaxone & $42(42$ j IV) \\
\hline $20 / \mathrm{H}$ & NS ; NS & Inconnue & Culture $+;$ S. moniliformis & $\begin{array}{l}\text { Fièvre, éruption cutanée, } \\
\text { polyarthralgie, dorsalgie }\end{array}$ & Ceftriaxone, pénicilline & $14(7 \mathrm{j}$ IV) \\
\hline $21 / F$ & $+;$ morsure & Inconnue & Culture $+;$ S. moniliformis & $\begin{array}{l}\text { Fièvre, épanchement } \\
\text { articulaire }\end{array}$ & Ceftriaxone, pénicilline & $11(4 \mathrm{j} I \mathrm{~V})$ \\
\hline $21 / H$ & $+; \mathrm{NS}$ & Inconnue & Culture + ; S. moniliformis & Fièvre, myalgie & Doxycycline & $14(0 \mathrm{j} I \mathrm{~V})$ \\
\hline $28 / F$ & + ; NS & Inconnue & Culture $+;$ S. moniliformis & $\begin{array}{l}\text { Fièvre, éruption cutanée, } \\
\text { polyarthralgie, vomissements, } \\
\text { diarrhée, pharyngite }\end{array}$ & Pénicilline & $21(3 \mathrm{j} I \mathrm{~V})$ \\
\hline $30 / F$ & + ; griffure & 8 jours & Culture +; S. moniliformis & $\begin{array}{l}\text { Fièvre, polyarthralgie, } \\
\text { vomissements, maux de tête, } \\
\text { érythème noueux soupçonné }\end{array}$ & Ceftriaxone & $28(28 \mathrm{j}$ IV $)$ \\
\hline $57 / \mathrm{H}$ & $+;$ morsure & 19 jours & $\begin{array}{l}\text { Culture -, ARN 16S +; S. } \\
\text { moniliformis }\end{array}$ & Fièvre, frissons & Ceftriaxone, doxycycline & $38(28$ j IV $)$ \\
\hline
\end{tabular}

Abréviations : ARN, acide ribonucléique; F, femme; H, homme; J-jour; IV, intraveineux; NS, non signalée; S. moniliformis, Streptobacillus moniliformis; -, négatif; +, positif;

Exposition à des rats domestiques dans tous les cas

${ }^{b}$ Durée du traitement intraveineux entre parenthèses

hémocultures positives, les flacons d'hémoculture se sont révélés positifs au cours des 24 heures d'incubation, et les sous-cultures réalisées en milieu solide ont démontré une croissance localisée de colonies dans les 48 heures. S. moniliformis a été identifié par spectrométrie de masse à temps de vol par désorption-ionisation laser assistée par matrice (MALDI-TOF; Bruker Daltonics,

Billerica, Massachusetts) dans ces quatre cas. Une épreuve de sensibilité non normalisée a révélé que les micro-organismes de ces quatre cas étaient sensibles à la pénicilline et à la ceftriaxone.

Sept cas ont nécessité une hospitalisation; la durée médiane du séjour était de cinq jours, variant de trois à 17 jours. Les schémas thérapeutiques complets ont été documentés pour tous les cas à l'exception d'un seul, dont on connaissait l'antibiotique, mais pas la durée du traitement (tableau 1). Dix cas (91\%) ont reçu des bêta-lactamines par voie intraveineuse comme traitement initial, la ceftriaxone étant l'antibiotique le plus souvent utilisé en consultation interne et externe. Le cas restant a reçu de la doxycycline en monothérapie, par voie orale, en partie en raison de l'allergie documentée du patient à la pénicilline. Dans neuf cas (82\%), des antibiotiques individuels ont été utilisés, tandis que dans deux cas (18\%), une association d'antibiotiques a été utilisée comme traitement empirique avant un ajustement en fonction des sensibilités. La durée totale médiane et moyenne de I'antibiothérapie était de 14 jours, mais variait de sept à 42 jours. Chez tous les patients traités par antibiothérapie, le traitement comprenait des antibiotiques en sevices externes par voie intraveineuse ou par voie orale à dose décroissante.

\section{Discussion}

Il s'agit de la série de cas de FMR la plus importante publiée au Canada. Le comté de San Diego, qui comptait 3095313 habitants en 2010, a enregistré 17 cas de FMR entre 2000 et 2012 (23). Fait intéressant, sur l'île de Vancouver, qui comptait une seulement 746058 habitants en 2010, 11 cas ont été documentés entre 2010 et 2016. Ainsi, l'incidence annuelle de la FMR corrigée en fonction de la population sur l'île de Vancouver était de 1,34 par million d'habitants par année, comparativement à 0,42 par million d'habitants par année à San Diego.

Plusieurs raisons peuvent expliquer la possiblitité d'incidence plus élevée sur l'île de Vancouver. En effet, la prévalence des rongeurs domestiques peut être plus élevée sur l'île de Vancouver, augmentant ainsi l'exposition aux rongeurs. II est possible que, compte tenu de la grande variabilité de la colonisation des muqueuses par S. moniliformis (3), sa prévalence dans la flore commensale des rats pourrait être plus élevée sur l'île de Vancouver. Par ailleurs, le fait que toutes les données pertinentes de la région soient centralisées dans le système Enterprise Data Warehouse d'Island Health peut avoir favorisé un repérage de cas plus exhaustif.

Sur l'île de Vancouver, S. moniliformis est le seul agent pathogène en cause dans les cas de FMR. Dans cette série de cas, la fièvre était présente dans tous les cas documentés; cependant, les autres symptômes classiques de la FMR, soit les éruptions cutanées et la polyarthralgie, n'ont été documentés que dans trois $(27 \%)$ et cinq $(45 \%)$ cas, respectivement. Après la fièvre et la polyarthralgie, les vomissements étaient le troisième symptôme le plus courant, touchant quatre $(36$ \%) cas. Les 
signes et symptômes restants, soit la myalgie, les céphalées et la pharyngite, ont été observés chez moins d'un tiers des cas, ce qui correspond aux observations d'une méta-analyse antérieure de signalement de cas (4). D'autres signes et symptômes, comme l'épanchement articulaire, la dorsalgie, les frissons et la diarrhée, ont également été documentés.

Malgré la forte sensibilité de S. moniliformis à de nombreuses classes d'antibiotiques, le traitement actuellement recommandé pour la FMR est la pénicilline $(3,15)$. Dans cette série de cas, la ceftriaxone était l'antibiotique empirique le plus souvent choisi et a fait partie du traitement dans huit (73 \%) cas; cependant, la pénicilline était le traitement à dose décroissante le plus courant (tableau 1). D'autres traitements, dont la gentamicine, la doxycycline et l'amoxicilline, ont été utilisés sans entraîner de complications. Fait important, un patient a répondu à la doxycycline administrée en monothérapie par voie orale pendant 14 jours.

La FMR est associée à un taux de mortalité d'environ $10 \%$ chez les personnes non traitées $(7,10,24)$. Par contre, aucun décès n'a été observé dans cette série de cas, car un traitement a été instauré rapidement dans tous les cas. De même, des études précédemment publiées ont montré que l'issue de la FMR était favorable quand un traitement était entrepris, et aucun cas de mortalité ou de morbidité à long terme n'a été observé. Cela vient davantage étayer l'idée selon laquelle la sensibilisation, le dépistage et l'administration judicieuse d'une antibiothérapie contribuent grandement à l'obtention d'issuespositives pour les patients.

Cette étude rétrospective comporte plusieurs limites liées à la portée de l'information et aux détails. Malgré une recherche exhaustive des cas de FMR dans la base de données d'Island Health, des cas ont pu être omis: patients ayant reçu un diagnostic en consultation externe et dont les hémocultures se sont révélées négatives ou n'ont pas été effectuées; patients de l'île de Vancouver dont le diagnostic et la prise en charge ont eu lieu à l'extérieur de l'île; et patients dont les détails sur l'exposition à des rongeurs n'ont pas été consignés. À l'avenir, des efforts devraient être déployés afin d'estimer le risque de FMR à la suite d'une griffure, d'une morsure ou d'un autre type d'exposition significative à la salive, à l'urine ou aux excréments de rats. Dans un cas, on constaté que le patient avait été mordu et griffé par son rat domestique à plusieurs reprises au cours des mois précédents avant de contracter la FMR. Les prochaines étapes de la recherche sur la FMR devraient comprendre une analyse à l'échelle provinciale visant à déterminer si les taux de FMR sur l'île de Vancouver sont supérieurs à ceux observés dans le reste de la Colombie-Britannique, car cela pourrait indiquer des lacunes dans le dépistage de la FMR ailleurs dans la province ou la présence d'un ou plusieurs facteurs locaux contribuant à une prévalence plus élevée sur l'île de Vancouver.

\section{Conclusion}

Cette étude constitue la plus grande série de cas de FMR observée à ce jour au Canada. Elle indique également une incidence plus élevée que prévu de FMR sur l'île de Vancouver. La FMR est une maladie rare, mis possiblement sous-déclarée en raison d'un tableau clinique non spécifique et des difficultés liées au diagnostic en laboratoire. Pour détecter la FMR, un indice de suspicion élevé est nécessaire afin de favoriser l'obtention de renseignements sur l'exposition d'un patient à des rongeurs lorsqu'il présente une fièvre avec éruptions cutanées, une polyarthralgie, des vomissements, une myalgie ou un épanchement articulaire. Une hémoculture est requise pour confirmer le diagnostic.

La FMR demeure une infection grave et peu reconnue. D'autres études permettraient de dresser un portrait précis de l'épidémiologie de la FMR au Canada.

\section{Déclaration des auteurs}

Les auteurs ont contribué en parts égales à ce manuscrit :

B. H. - Conceptualisation, méthodologie, recherche, rédaction ébauche initiale, examen et révision

C. W. - Conceptualisation, méthodologie, recherche, rédaction ébauche initiale, examen et révision

K. T. - Conceptualisation, méthodologie, ressources, rédaction examen et révision

Les opinions exprimées dans l'article soumis sont celles des auteurs, et non la position officielle de l'Université de la Colombie-Britannique.

\section{Conflit d'intérêts}

Aucun.

\section{Remerciements}

Nous tenons à remercier les cliniciens et les équipes de professionnels paramédicaux qui ont participé aux soins des patients infectés par la FMR. Nous tenons également à remercier Rebecca Raworth, bibliothécaire, Island Medical Program, pour son soutien dans le processus d'examen de la documentation.

\section{Financement}

Aucun.

\section{Références}

1. Dendle C, Woolley IJ, Korman TM. Rat-bite fever septic arthritis: illustrative case and literature review. Eur J Clin Microbiol Infect Dis 2006 Dec;25(12):791-7. https://doi. org/10.1007/s10096-006-0224-x. PubMed (https://www.ncbi. nlm.nih.gov/pubmed/17096137?dopt=Abstract)

2. Suzuki $K$, Hirai Y, Morita F, Nakamura A, Uehara Y, Naito T. Streptobacillus moniliformis Bacteremia in a Pet Shop Employee: Case Report and Literature Review. Open Forum Infect Dis 2017 Mar;4(2):ofx038. https://doi.org/10.1093/ ofid/ofx038. PubMed (https://www.ncbi.nlm.nih.gov/ pubmed/28730157?dopt=Abstract) 
14. Kondruweit M, Weyand M, Mahmoud FO, Geissdörfer W, Schoerner C, Ropers D, Achenbach S, Strecker T. Fulminant endocarditis caused by Streptobacillus moniliformis in a young man. J Thorac Cardiovasc Surg 2007 Dec;134(6):1579-80. https://doi.org/10.1016/j.jtcvs.2007.08.010. PubMed (https:// www.ncbi.nlm.nih.gov/pubmed/18023687?dopt=Abstract)

4. Elliott SP. Rat bite fever and Streptobacillus moniliformis. Clin Microbiol Rev 2007 Jan;20(1):13-22. https://doi.org/10.1128/ CMR.00016-06. PubMed (https://www.ncbi.nlm.nih.gov/ pubmed/17223620?dopt=Abstract)

5. Graves MH, Janda JM. Rat-bite fever (Streptobacillus moniliformis): a potential emerging disease. Int J Infect Dis 2001;5(3):151-5. https://doi.org/10.1016/S12019712(01)90090-6. PubMed (https://www.ncbi.nlm.nih.gov/ pubmed/11724672?dopt=Abstract)

6. Hirschhorn RB, Hodge RR. Identification of risk factors in rat bite incidents involving humans. Pediatrics 1999 Sep;104(3):e35. https://doi.org/10.1542/ peds.104.3.e35. PubMed (https://www.ncbi.nlm.nih.gov/ pubmed/10469818?dopt=Abstract)

7. Centers for Disease Control and Prevention (CDC). Fatal rat-bite fever--Florida and Washington, 2003. MMWR Morb Mortal Wkly Rep 2005 Jan;53(51):1198-202. PubMed (https:// www.ncbi.nlm.nih.gov/pubmed/15635289?dopt=Abstract)

8. Ordog GJ, Balasubramanium S, Wasserberger J. Rat bites: fifty cases. Ann Emerg Med 1985 Feb;14(2):126-30. https://doi. org/10.1016/S0196-0644(85)81073-8. PubMed (https://www. ncbi.nlm.nih.gov/pubmed/3970397?dopt=Abstract)

9. Eisenberg T, Ewers C, Rau J, Akimkin V, Nicklas W. Approved and novel strategies in diagnostics of rat bite fever and other Streptobacillus infections in humans and animals. Virulence 2016 Aug;7(6):630-48. https://doi.org/10.1080/2150559 4.2016.1177694. PubMed (https://www.ncbi.nlm.nih.gov/ pubmed/27088660?dopt=Abstract)

10. Ojukwu IC, Christy C. Rat-bite fever in children: case report and review. Scand J Infect Dis 2002;34(6):474-7. https://doi. org/10.1080/003655402320170345. PubMed (https://www. ncbi.nlm.nih.gov/pubmed/12160180?dopt=Abstract)

11. Carbeck RB, Murphy JF, Britt EM. Streptobacillary rat-bite fever with massive pericardial effusion. JAMA 1967 Aug;201(9):703-4. https://doi.org/10.1001/ jama.1967.03130090067024. PubMed (https://www.ncbi.nlm. nih.gov/pubmed/5340333?dopt=Abstract)

12. Faro S, Walker C, Pierson RL. Amnionitis with intact amniotic membranes involving Streptobacillus moniliformis. Obstet Gynecol 1980 Mar;55(3 Suppl):9S-11S. https://doi. org/10.1097/00006250-198003001-00003. PubMed (https:// www.ncbi.nlm.nih.gov/pubmed/7360458?dopt=Abstract)

13. Tattersall RS, Bourne JT. Systemic vasculitis following an unreported rat bite. Ann Rheum Dis 2003 Jul;62(7):605-6. https://doi.org/10.1136/ard.62.7.605. PubMed (https://www. ncbi.nlm.nih.gov/pubmed/12810419?dopt=Abstract) 


\section{RMTC \\ LA FIÈVRE PAR MORSURE DE RAT (FMR) DANS L'ÎLE DE \\ VANCOUVER : UNE MALADIE RARE MAIS PLUS FRÉQUENTE QUE PRÉVU}

\section{La FMR est rare}

La FMR est une infection peu commune causée par des bactéries faisant partie de la flore normale de la gueule des rongeurs (rats, souris, gerbilles, etc.)

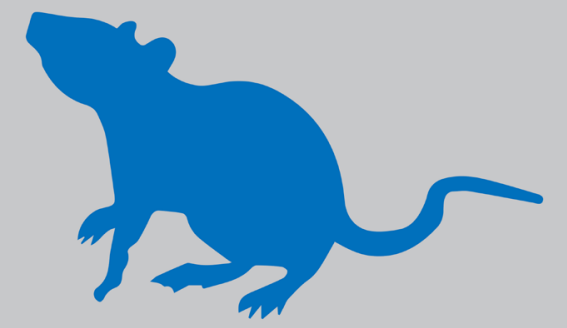

On la contracte en par morsure ou égratignure d'un rongeur ou en entrant en contact avec son urine ou ses excréments. On compte parmi ses symptômes la fièvre, des frissons, des éruptions cutanées et des douleurs musculaires ou articulaires.

\section{Plus de cas que prévu}

On comptait 11 cas dans l'île de Vancouver entre 2010 et $2016^{\star}$. Sept cas ont été hospitalisés.

Étant donné que plus de gens gardent des rongeurs apprivoisés, l'exposition à la FMR et le risque de l'attraper ont augmenté.

\section{On peut traiter et prévenir la FMR}

\section{TRAITEMENT}

On peut facilement traiter la FMR avec des antibiotiques. Par contre, elle peut devenir une maladie grave si on ne la traite pas.

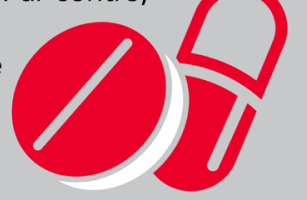

\section{PRÉVENTION}

Si vous avez un rongeur apprivoisé, lavez-vous bien les mains après l'avoir manipulé. Si vous présentez des symptômes après qu'il

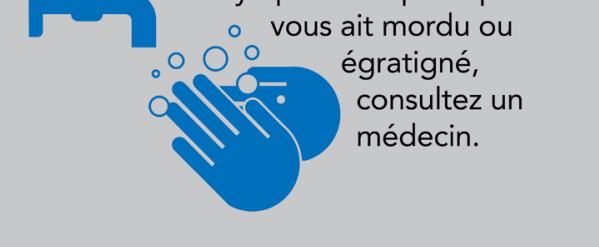

